Bull. Korean Math. Soc. 51 (2014), No. 1, pp. 157-171

http://dx.doi.org/10.4134/BKMS.2014.51.1.157

\title{
METRIC THEOREM AND HAUSDORFF DIMENSION ON RECURRENCE RATE OF LAURENT SERIES
}

\author{
Xue-hai Hu, Bing Li, And Jian Xu
}

\begin{abstract}
We show that the recurrence rates of Laurent series about continued fractions almost surely coincide with their pointwise dimensions of the Haar measure. Moreover, let $E_{\alpha, \beta}$ be the set of points with lower and upper recurrence rates $\alpha, \beta(0 \leq \alpha \leq \beta \leq \infty)$, we prove that all the sets $E_{\alpha, \beta}$ are of full Hausdorff dimension. Then the recurrence sets $E_{\alpha, \beta}$ have constant multifractal spectra.
\end{abstract}

\section{Introduction}

Let $\mathbb{F}_{q}$ be a finite field of $q$ elements and $\mathbb{F}_{q}\left(\left(z^{-1}\right)\right)$ denote the field of all formal Laurent series with coefficients in $\mathbb{F}_{q}$, and $\mathbb{F}_{q}[z]$ denote the ring of polynomials with coefficients in $\mathbb{F}_{q}$.

For each $x=\sum_{n=n_{0}}^{\infty} c_{n} z^{-n} \in \mathbb{F}_{q}\left(\left(z^{-1}\right)\right)$ with $n_{0} \in \mathbb{Z}$, denote

$$
[x]=\sum_{n=n_{0}}^{0} c_{n} z^{-n} \in \mathbb{F}_{q}[z],
$$

which is called the integer part of $x$, and the order of $x$ is defined as $v(x)=$ $-\operatorname{deg}(x)=\inf \left\{n \in \mathbb{Z}: c_{n} \neq 0\right\}$. Define a non-Archimedean valuation on $\mathbb{F}_{q}\left(\left(z^{-1}\right)\right)$ as $\|x\|=q^{-v(x)}$ for all $x \in \mathbb{F}_{q}\left(\left(z^{-1}\right)\right)$. The field $\mathbb{F}_{q}\left(\left(z^{-1}\right)\right)$ is locally compact and complete under the metric $\rho\left(x, x^{\prime}\right)=\left\|x-x^{\prime}\right\|$.

Remark 1.1. Since the valuation $\|\cdot\|$ is non-Archimedean, it follows that if two discs intersect, then one contains the other.

Let $I$ denote the valuation ideal of $\mathbb{F}_{q}\left(\left(z^{-1}\right)\right)$, i.e.,

$$
I=\left\{x \in \mathbb{F}_{q}\left(\left(z^{-1}\right)\right):\|x\|<1\right\}=\left\{x=\sum_{n=1}^{\infty} c_{n} z^{-n}: c_{n} \in \mathbb{F}_{q}\right\}
$$

Received January 16, 2013; Revised May 16, 2013

2010 Mathematics Subject Classification. 28A80.

Key words and phrases. recurrence rate, pointwise dimension, continued fractions, Laurent series, Hausdorff dimension. 
and $P$ the Haar measure on $\mathbb{F}_{q}\left(\left(z^{-1}\right)\right)$ normalized to 1 on $I$. For any $x \in$ $\mathbb{F}_{q}\left(\left(z^{-1}\right)\right)$ and $m \in \mathbb{Z}$, let

$$
B\left(x, q^{-m}\right)=\left\{y \in \mathbb{F}_{q}\left(\left(z^{-1}\right)\right):\|y-x\|<q^{-m}\right\}
$$

be the open disc of center $x$ and radius $q^{-m}$, we have $P\left(B\left(x, q^{-m}\right)\right)=q^{-m}$.

Consider the following transformation from $I$ to $I$ defined by

$$
T x:=\frac{1}{x}-\left[\frac{1}{x}\right], \quad T 0:=0 .
$$

This map describes the regular continued fraction over the field of Laurent series and has been introduced by Artin [1]. As in the classical theory, every $x \in I$ has the following continued fraction expansion:

$$
x=\frac{1}{A_{1}(x)+\frac{1}{A_{2}(x)+\frac{1}{A_{3}(x)+\cdots}}}:=\left[A_{1}(x), A_{2}(x), A_{3}(x), \ldots\right],
$$

where the digits $A_{n}(x)$ are polynomials of strictly positive degree and are defined by $A_{n}(x)=\left[\frac{1}{T^{n-1}(x)}\right], \forall n \geq 1$. We denote by $\mathfrak{D}$ the set of polynomials with strictly positive degree. The sequence of random variables $\left\{\operatorname{deg} A_{k}(\cdot)\right\}$ is independent and identically distributed with respect to $P$ (see also Berthé and Nakada [4], Paysant-Leroux and Dubois [13]).

The metric and ergodic properties of this dynamical system have been studied extensively (see, e.g. Schmidt [15], Berthé and Nakada [4]). For results on the dimension theory, we refer to Niederreiter and Vielhaber [10, 11], Wu [16], $\mathrm{Hu}$, Wang, Wu and $\mathrm{Yu}[8]$.

The main motivation for this work is the quantitative behavior of Poincaré recurrence. Let $(X, \mathscr{B}, T, \mu)$ be an abstract measure preserving dynamical system. The famous Poincaré Recurrence Theorem states that if $A$ is a measurable set of the space with positive measure, then the orbit of $x$ returns to $A$ infinitely many times for $\mu$-almost every $x \in A$.

In the past decade, lots of authors paid more attention to this area. Firstly, Boshernitzan [5] said that if the space $X$ is separable and the Hausdorff measure $H_{\alpha}(X)$ is $\sigma$-finite for some $\alpha>0$, then

$$
\liminf _{n \rightarrow \infty} n^{1 / \alpha} d\left(T^{n} x, x\right)<\infty, \quad \mu \text {-almost everywhere (a.e. for short). }
$$

Define the first return time of a point $x \in X$ into the set $A$ by

$$
\tau_{A}(x)=\inf \left\{k \in \mathbb{N}: T^{k} x \in A\right\} .
$$

Ornstein and Weiss [12] proved that for a finite partition $\xi$, if there exists a $T$-invariant ergodic Borel probability measure $\mu$, then

$$
\lim _{n \rightarrow \infty} \frac{\log \tau_{\xi_{n}(x)}(x)}{n}=h_{\mu}(\xi) \quad \mu \text {-a.e. }
$$


where $\xi_{n}(x)$ is the intersection of $\xi, T^{-1}(\xi), \ldots, T^{-n+1}(\xi)$ which contains $x$, and $h_{\mu}(\xi)$ denotes the measure-theoretic entropy of $T$ with respect to the partition $\xi$. Moreover, in the special case $X \subset \mathbb{R}^{d}$ for some $d \in \mathbb{N}$, Barreria and Saussol [2] strengthened the above results by proving that

$$
\tau_{r}(x):=\inf \left\{n \geq 1: T^{n} x \in B(x, r)\right\} \sim r^{-d_{\mu}}, \quad \mu \text {-a.e., }
$$

where $d_{\mu}$ is the Hausdorff dimension of the measure.

More precisely, define the lower and upper recurrence rate of $x$ by

$$
\underline{R}(x)=\liminf _{r \rightarrow 0} \frac{\log \tau_{r}(x)}{-\log r}, \quad \bar{R}(x)=\limsup _{r \rightarrow 0} \frac{\log \tau_{r}(x)}{-\log r} .
$$

If two numbers $\underline{R}(x)$ and $\bar{R}(x)$ are same, then their common value is denoted by $R(x)$, called the recurrence rate of $x$. On the other hand, the lower and upper pointwise dimensions of $\mu$ at a point $x \in X$ are given by

$$
\underline{d}_{\mu}(x)=\liminf _{r \rightarrow 0} \frac{\log \mu(B(x, r))}{\log r}, \quad \bar{d}_{\mu}(x)=\liminf _{r \rightarrow 0} \frac{\log \mu(B(x, r))}{\log r} .
$$

With the above notations, for $X \subset \mathbb{R}^{d}$, Barreria and Saussol [2] proved that

$$
\underline{R}(x)=\underline{d}_{\mu}(x), \quad \bar{R}(x)=\bar{d}_{\mu}(x), \quad \mu \text {-a.e. }
$$

with the conditions that $\mu$ has long return time (see [2]) and $\underline{d}_{\mu}(x)>0$ for $\mu$ a.e.x. A simple consequence of this result is a reformulation of Boshernitzan's result

holds for all $\alpha>\underline{d}_{\mu}(x)$.

$$
\liminf _{n \rightarrow \infty} n^{1 / \alpha} d\left(T^{n} x, x\right)=0
$$

Following in time, many researchers (for example, [3]) studied the problem when the formulation (1) holds from many different points of view. Barreria and Saussol [3] proved that the formulation (1) holds for three important different situations, including repellers of $C^{1+\alpha}$ expanding maps together with equilibrium measures of Hölder continuous functions and the continued fraction transformation endowed Gauss measure.

In this paper, we study the quantitative recurrence rate of the measure preserving dynamical system $(I, \mathscr{B}(I), T, P)$ and show that the formulation (1) holds for this system. We will show that each recurrence rate is one for almost all points in $I$. We remark that the former results $[2,3]$ can not cover our case.

Theorem 1.2. For $P$-almost all $x \in I$, we have

$$
R(x)=1 .
$$

Moreover, we calculate the Hausdorff dimensions of level sets related to Theorem 1.2 and prove that these kind of sets have constant multifractal spectra.

Theorem 1.3. Let $\alpha, \beta$ be two real numbers with $0 \leq \alpha \leq \beta \leq+\infty$. Then the following set

$$
E_{\alpha, \beta}=\{x \in I: \underline{R}(x)=\alpha, \bar{R}(x)=\beta\}
$$


has Hausdorff dimension one.

In other cases, the Hausdorff dimensions of the sets like (2) have been considered by many different authors. Feng and Wu [7] established the result in symbolic space with finite symbols. Saussol and Wu [14] studied the case of conformal repeller of the $C^{1+\alpha}$ map. Recently, Lau and Shu [9] got a similar result for the topological entropy of the set $E_{\alpha, \beta}$ under the conditions of positively expansive and specification condition with the Bowen metric.

\section{Basic properties}

In this section, we collect a few results about the continued fraction expansion of Laurent series which we use frequently (see Niederreiter [10] or Berthé and Nakada [4]).

Let $x \in I$ and $\left\{\frac{P_{n}(x)}{Q_{n}(x)}, n \geq 1\right\}$ be the sequence of convergents of its continued fraction expansion, i.e.,

$$
\frac{P_{n}(x)}{Q_{n}(x)}:=\left[A_{1}(x), \ldots, A_{n}(x)\right] .
$$

Proposition $2.1([4,10,11])$. Let $x \in I$. Then we have

(1) $x=\sum_{k=1}^{\infty} \frac{(-1)^{k}}{Q_{k}(x) Q_{k+1}(x)}, \quad \frac{P_{n}(x)}{Q_{n}(x)}=\sum_{k=1}^{n} \frac{(-1)^{k}}{Q_{k}(x) Q_{k+1}(x)}$.

(2) $\left\|Q_{n}(x)\right\|=\prod_{k=1}^{n}\left\|A_{k}(x)\right\|$.

(3) $\left\|x-\frac{P_{n}(x)}{Q_{n}(x)}\right\|=\frac{1}{\left\|Q_{n}(x) Q_{n+1}(x)\right\|}=\frac{1}{\left\|A_{n+1}(x) Q_{n}(x)^{2}\right\|}<\frac{1}{\left\|Q_{n}\right\|^{2}}$.

Lemma $2.2([4,10])$. For any $A_{1}, A_{2}, \ldots, A_{n} \in \mathfrak{D}$, we call

$$
I\left(A_{1}, \ldots, A_{n}\right)=\left\{x \in I: A_{1}(x)=A_{1}, \ldots, A_{n}(x)=A_{n}\right\}
$$

an $n$-th order fundamental cylinder. Then the set $I\left(A_{1}, A_{2}, \ldots, A_{n}\right)$ is a disc with diameter

$$
\left|I\left(A_{1}, A_{2}, \ldots, A_{n}\right)\right|=q^{-2 \sum_{k=1}^{n} \operatorname{deg} A_{k}-1}
$$

and

$$
P\left(I\left(A_{1}, A_{2}, \ldots, A_{n}\right)\right)=q^{-2 \sum_{k=1}^{n} \operatorname{deg} A_{k}},
$$

where $P$ is the Haar measure on $I$.

\section{Metric theorem about recurrent rate}

In this section, we will show some lemmas firstly, which induce Theorem 1.2 directly.

For any $r>0$, we can find $n \in \mathbb{N}$ such that $q^{-(n+1)}<r \leq q^{-n}$, then

$$
\underline{R}(x)=\liminf _{n \rightarrow \infty} \frac{\log _{q} \tau_{q^{-n}}(x)}{n} \text { and } \bar{R}(x)=\limsup _{n \rightarrow \infty} \frac{\log _{q} \tau_{q^{-n}}(x)}{n} .
$$


So we only need to consider the limits like (3) as $n \rightarrow \infty$ when referring to the lower and upper recurrent rate.

In order to prove Theorem 1.2, we only need to show that, for $P$-almost every $x \in I$, we both have $\underline{R}(x) \geq 1$ and $\bar{R}(x) \leq 1$.

Lemma 3.1. The whole space I can be partitioned to $q^{m}$ disjoint balls with radius $q^{-m}$, that is, there exist $y_{1}, \ldots, y_{q^{m}} \in I$ such that

$$
I=\bigsqcup_{i=1}^{q^{m}} B\left(y_{i}, q^{-m}\right)
$$

Proof. Since $\left\{B\left(y, q^{-m}\right)\right\}_{y \in I}$ is a open cover of $I$ and $I$ is compact, there exists a finite set $\left\{y_{1}, y_{2}, \ldots, y_{k}\right\}$ such that $I \subset \cup_{i=1}^{k} B\left(y_{i}, q^{-m}\right)$. By Remark 1.1, we can choose $B\left(y_{i}, q^{-m}\right) \cap B\left(y_{j}, q^{-m}\right)=\emptyset$ for $i \neq j$. Then

$$
I=\sqcup_{i=1}^{k} B\left(y_{i}, q^{-m}\right) \text {. }
$$

Since $P(I)=1$ and $\left\{B\left(y_{i}, q^{-m}\right)\right\}$ are mutually disjoint, then $k=q^{m}$.

The first return time of the point $y \in B(x, r)$ into $B(x, r)$ is defined by

$$
\tau_{r}(y, x):=\inf \left\{k \geq 1: d\left(T^{k} y, x\right)<r\right\} .
$$

Remark 3.2. If $\|x-y\|<q^{-m}$, then

$$
\tau_{q^{-m}}(x)=\tau_{q^{-m}}(y)=\tau_{q^{-m}}(y, x) .
$$

Lemma 3.3. For P-almost all $x \in I$, we have

$$
\bar{R}(x) \leq 1 \text {. }
$$

Proof. For any $\epsilon>0$, let $D_{m}(\epsilon)=\left\{x \in I: \tau_{q^{-m}}(x)>q^{m(1+\epsilon)}\right\}$. By BorelCantelli lemma, we are led to prove that $\sum_{m=1}^{\infty} P\left(D_{m}(\epsilon)\right)<+\infty$.

In fact, by Lemma 3.1, we have the partition (4), then

$$
P\left(D_{m}(\epsilon)\right)=\sum_{i=1}^{q^{m}} P\left\{x \in B\left(y_{i}, q^{-m}\right): \tau_{q^{-m}}(x)>q^{m(1+\epsilon)}\right\} .
$$

Chebyshev's inequality implies

(7) $P\left\{x \in B\left(y, q^{-m}\right): \tau_{q^{-m}}(x, y)>q^{m(1+\epsilon)}\right\} \leq \frac{\int_{B\left(y, q^{-m}\right)} \tau_{q^{-m}}(x, y) d P(x)}{q^{m(1+\epsilon)}}$.

By Kac's theorem, we know that for any $y \in I$,

$$
\int_{B\left(y, q^{-m}\right)} \tau_{q^{-m}(x, y) d P(x)=1 .}
$$

By Remark 3.2, we have (5). Combining (5), (7) and (8), we have

$$
P\left\{x \in B\left(y, q^{-m}\right): \tau_{q^{-m}}(x)>q^{m(1+\epsilon)}\right\} \leq q^{-m(1+\epsilon)} .
$$

Therefore, by (6) and (9), we know $P\left(D_{m}(\epsilon)\right) \leq \sum_{i=1}^{q^{m}} q^{-m(1+\epsilon)} \leq q^{-m \epsilon}$, which implies the convergence of the series $\sum_{m=1}^{\infty} P\left(D_{m}(\epsilon)\right)$. 
For the inverse inequality, we note that the equalities $\underline{R}(x)=\underline{d}_{\mu}(x), \bar{R}(x)=$ $\bar{d}_{\mu}(x), \mu$-a.e. in [2] require the important property named long return time on the measure $\mu$. Let us recall some notations related to long return time. Let $(X, \mathcal{B}, \mu, T)$ be a measure-preserving dynamical system and $x \in X, r, \epsilon>0$. Consider the set

$$
E_{\epsilon}(x, r)=\left\{y \in B(x, r): \tau_{r}(y, x) \leq \mu(B(x, r))^{-1+\epsilon}\right\} .
$$

We say that the measure $\mu$ has long return time if

$$
\liminf _{r \rightarrow 0} \frac{\log \mu\left(E_{\epsilon}(x, r)\right)}{\log \mu(B(x, r))}>1
$$

for $\mu$-almost every $x \in X$ and every sufficiently small $\epsilon>0$.

In the sequel, we shall verify that the dynamical system $(I, \mathscr{B}(I), T, P)$ satisfies the property long return time.

Lemma 3.4. The dynamical system $(I, \mathscr{B}(I), T, P)$ satisfies the property of long return time.

Proof. For any $x \in I$ and $r>0$, there exists $n \in \mathbb{N}$ such that $q^{-(n+1)}<r \leq$ $q^{-n}$, we put

and

$$
\begin{gathered}
m_{1}=\max \left\{m \geq 0: 2 \sum_{k=1}^{m} \operatorname{deg} A_{k}(x) \leq n\right\}, \\
p_{1}=\sum_{k=1}^{m_{1}} \operatorname{deg} A_{k}(x), \quad p_{2}=\sum_{k=1}^{m_{1}+1} \operatorname{deg} A_{k}(x),
\end{gathered}
$$

$$
\widetilde{E}_{\varepsilon}(x, r)=\left\{y \in B\left(x, q^{-2 p_{1}}\right): \tau_{q^{-2 p_{1}}}(y, x) \leq P\left(B\left(x, q^{-2 p_{2}}\right)\right)^{-1+\varepsilon}\right\} .
$$

Here $E_{\varepsilon}(x, r) \subset \widetilde{E}_{\varepsilon}(x, r)$. Note that $B\left(x, q^{-2 p_{1}}\right)=I\left(A_{1}(x), \ldots, A_{m_{1}}(x)\right)$ and $\left\{A_{k}(\cdot)\right\}$ is a sequence of i.i.d. random variables with respect to $P$, by simple calculation, we have

$$
P\left(\bigcup_{A_{1}, \ldots, A_{m} \in \mathfrak{D}}\left\{I\left(A_{1}, \ldots, A_{m}\right) \cap T^{-k} I\left(A_{1}, \ldots, A_{m}\right)\right\}\right) \leq q^{-2 m}
$$

for $1 \leq k<m$ and

$$
P\left(\bigcup_{k=1}^{m-1} \bigcup_{A_{1}, \ldots, A_{m} \in \mathfrak{D}}\left\{I\left(A_{1}, \ldots, A_{m}\right) \cap T^{-k} I\left(A_{1}, \ldots, A_{m}\right)\right\}\right) \leq \frac{m-1}{q^{2 m}} .
$$

Then by Borel-Cantelli Lemma, it turns out that for $P$-a.e. $x$ (denote by $U$ such set of full measure),

$$
\tau_{q^{-2 p_{1}}}(y, x) \geq m_{1}
$$

for sufficient small $r>0$. For any $x \in U$, we know

$$
\widetilde{E}_{\varepsilon}(x, r) \subset \bigcup_{k=m_{1}}^{\left[P\left(B\left(x, q^{-2 p_{2}}\right)\right)^{-1+\varepsilon}\right]} I\left(A_{1}, \ldots, A_{m_{1}}\right) \cap T^{-k} I\left(A_{1}, \ldots, A_{m_{1}}\right),
$$


it turns out that

$$
\begin{aligned}
P\left(E_{\varepsilon}(x, r)\right) & \leq P\left(\widetilde{E}_{\varepsilon}(x, r)\right) \\
& \leq\left\{P\left(B\left(x, q^{-2 p_{2}}\right)\right)^{-1+\varepsilon}-m_{1}\right\} q^{-2 p_{1}} q^{-2 p_{1}} \\
& \leq q^{-4 p_{1}} q^{2 p_{2}(1-\varepsilon)}
\end{aligned}
$$

for sufficient small $r>0$. Hence we have

$$
\frac{\log P\left(E_{\varepsilon}(x, r)\right)}{\log P(B(x, r))} \geq \frac{2 p_{1}-p_{2}}{p_{2}}+\varepsilon .
$$

For $P$-a.e. $x, \frac{p_{1}}{p_{2}} \rightarrow 1$ as $r \rightarrow 0$ due to the strong law of large numbers for $\left\{\operatorname{deg} A_{k}(\cdot)\right\}$, we know

$$
\liminf _{r \rightarrow 0} \frac{\log P\left(E_{\varepsilon}(x, r)\right)}{\log P(B(x, r))} \geq 1+\varepsilon .
$$

Thus we obtain the desired result.

Lemma 3.5. For P-almost every $x \in I$, we have

$$
\underline{R}(x) \geq 1 \text {. }
$$

Proof. For any $\epsilon>0$ and $m \in \mathbb{N}$, let

$$
H_{m}(\epsilon)=\left\{y \in I: \tau_{q^{-m}}(y)<q^{m(1-\epsilon)}\right\} .
$$

For any $x \in I$, if $y \in B\left(x, q^{-m}\right)$, then $\tau_{q^{-m}}(y)=\tau_{q^{-m}}(y, x)$ by Remark 3.2. Then

$$
\begin{aligned}
B\left(x, q^{-m}\right) \cap H_{m}(\epsilon) & =\left\{y \in B\left(x, q^{-m}\right): \tau_{q^{-m}}(y, x) \leq q^{m(1-\epsilon)}\right\} \\
& =E_{\epsilon}\left(x, q^{-m}\right) .
\end{aligned}
$$

Let $B\left(x_{1}, q^{-m}\right), B\left(x_{2}, q^{-m}\right), \ldots, B\left(x_{q^{m}}, q^{-m}\right)$ be a partition of $I$ (in the light of lemma 3.1). By Lemma 3.4, there exist $\kappa_{i}>0$ such that

$$
P\left(E_{\epsilon}\left(x_{i}, q^{-m}\right)\right) \leq q^{-m\left(1+\kappa_{i}\right)}\left(i=1, \ldots, q^{m}\right) .
$$

Let $\kappa=\min \left\{\kappa_{1}, \ldots, \kappa_{q^{m}}\right\}$. Then for each $i=1, \ldots, q^{m}$, we have

$$
P\left(B\left(x_{i}, q^{-m}\right) \cap H_{m}(\epsilon)\right) \leq q^{-m(1+\kappa)} .
$$

Then

$$
\begin{aligned}
P\left(H_{m}(\varepsilon)\right) & =P\left(\cup_{i=1}^{q^{m}} B\left(x_{i}, q^{-m}\right) \cap H_{m}(\epsilon)\right) \\
& =\sum_{i=1}^{q^{m}} P\left(B\left(x_{i}, q^{-m}\right) \cap H_{m}(\epsilon)\right) \\
& \leq q^{m} \cdot q^{-m(1+\kappa)}=q^{-m \kappa} .
\end{aligned}
$$

Therefore, $\sum_{m=1}^{\infty} P\left(H_{m}(\varepsilon)\right)<\infty$. Broel-Cantelli Lemma implies that for $P$ almost every $y \in I$, we have

$$
\tau_{q^{-m}}(y)>q^{m(1-\epsilon)} \text { when } m \text { is large enough. }
$$

Then $\underline{R}(x) \geq 1$ by the arbitrary of $\epsilon$. 
Proof of Theorem 1.2. Lemma 3.3 and Lemma 3.5 imply the result of Theorem 1.2 .

\section{Hausdorff dimensions of the sets $E_{\alpha, \beta}$}

In this section, we give the proof of Theorem 1.3. To make the argument clear, we decompose the proof to several steps. Firstly, we shall construct a Cantor-like subset of $E_{\alpha, \beta}$ with large dimension (nearly to 1).

\subsection{Constructing a Cantor set with large dimension}

Here we cite a result due to $\mathrm{Wu}[16]$, which will be used later.

Proposition 4.1. Let $S$ be a non-empty finite set of polynomials with strictly positive degree and coefficients lying in $\mathbb{F}_{q}$, say $S=\left\{a_{1}, \ldots, a_{m}\right\}$. Write

$$
E_{S}=\left\{x \in I: A_{i}(x) \in S \text { for } i \geq 1\right\} .
$$

Then $\operatorname{dim}_{H} E_{S}=t$, where $t$ is given by

$$
\sum_{k=1}^{m} q^{-2 t \operatorname{deg} a_{k}}=1 .
$$

For $M \geq 1$, define $F(M)=\left\{x \in I: \operatorname{deg} A_{i}(x) \leq M\right.$ for all $\left.i \geq 1\right\}$, by Proposition 4.1, the Hausdorff dimension of the set $F(M)$ is $t_{M}$, which is the unique solution to the equation

$$
\sum_{k=1}^{M}(q-1) q^{k} q^{-2 k t}=1 .
$$

\section{Proposition 4.2.}

$$
\lim _{M \rightarrow \infty} t_{M}=1 .
$$

Proof. The proof is elementary.

Let $p>2$ be an integer. Based on the set $F(M)$, we construct a Cantor subset of $F(M)$,

$$
\begin{aligned}
F(M, p)=\{x \in F(M): & \operatorname{deg} A_{1}(x)=\cdots=\operatorname{deg} A_{p}(x)=M \text { and } \\
& \left.\operatorname{deg} A_{k p+1}(x)=\operatorname{deg} A_{k p+p}(x)=1 \text { for all } k \geq 1\right\} .
\end{aligned}
$$

In order to obtain lower bound of Hausdorff dimensions, we need the following mass distribution principle (see [6, Proposition 4.2]).

Proposition 4.3 (Mass Distribution Principle). Suppose $E \subset I$ and $\mu$ is a measure with $\mu(E)>0$. If there exist constants $c>0$ and $\delta>0$ such that

$$
\mu(D) \leq c|D|^{s}
$$

for all discs $D$ with diameter $|D| \leq \delta$, then

$$
\operatorname{dim}_{H} E \geq s .
$$


We will show that $F(M, p)$ is a large dimensional subset of $F(M)$ by proving the following lemma.

Lemma 4.4. Let $\epsilon \geq 0$ satisfies the condition

$$
2 t_{M}-\log _{q} q(q-1)=\epsilon p \text {. }
$$

Then we have

$$
\operatorname{dim}_{H} F(M, p) \geq t_{M}-\epsilon .
$$

By the above result, when we choose $p$ large enough, we know $\operatorname{dim}_{H} F(M, p)$ will be close to $t_{M}$.

\section{Corollary 4.5.}

$$
\lim _{p \rightarrow \infty} \operatorname{dim}_{H} F(M, p)=t_{M} .
$$

Proof of Lemma 4.4. To get the lower bound of $\operatorname{dim}_{H} F(M, p)$, we will give a probability measure supported on it. In the light of mass distribution principle, we need to show that the given measure satisfies (12).

\section{Part I. The measure $\mu$ supported on $F(M, p)$}

In this part, we define a probability measure $\mu$ supported on $F(M, p)$. In part II and III we will show that the measure satisfies (12).

We will give the fractal structure of the set $F(M, p)$ as the following. For any $n \geq 1$, denote

$$
\begin{array}{r}
D_{n}=\left\{\left(A_{1}, \ldots, A_{n}\right) \in \mathfrak{D}^{n}: \operatorname{deg} A_{i} \leq M \text { for all } i \geq 1, \operatorname{deg} A_{1}=\cdots=\operatorname{deg} A_{p}\right. \\
\left.=M \text { and } \operatorname{deg} A_{k p+1}=\operatorname{deg} A_{k p+p}=1 \text { for } k \geq 1\right\} .
\end{array}
$$

For any $\left(A_{1}, \ldots, A_{n}\right) \in D_{n}$, call

$$
I\left(A_{1}, \ldots, A_{n}\right):=\left\{x \in I: A_{k}(x)=A_{k}, 1 \leq k \leq n\right\}
$$

an admissible cylinder of order $n$ (with respect to $F(M, p)$ ). Then it gives

$$
F(M, p)=\bigcap_{n=1}^{\infty} \bigcup_{\left(A_{1}, \ldots, A_{n}\right) \in D_{n}} I\left(A_{1}, \ldots, A_{n}\right) .
$$

Now a set function $\mu$ will be constructed on admissible cylinders as the following.

For $n=0, \mu(I)=1$.

For $1 \leq n \leq p$,

$$
\mu\left(I\left(A_{1}, \ldots, A_{n}\right)\right)=\frac{1}{(q-1) q^{M}} \mu\left(I\left(A_{1}, \ldots, A_{n-1}\right)\right) .
$$

For any $n>p$ and $\left(A_{1}, \ldots, A_{n}\right) \in D_{n}$ we define:

if $n=k p+1, k p+p$ for some $k \geq 1$, set

$$
\mu\left(I\left(A_{1}, \ldots, A_{n}\right)\right)=\frac{1}{(q-1) q} \mu\left(I\left(A_{1}, \ldots, A_{n-1}\right)\right) .
$$


if $n \neq k p+1, k p+p$ for some $k \geq 1$, set

$$
\mu\left(I\left(A_{1}, \cdots, A_{n}\right)\right)=\frac{1}{\left(q^{2 \operatorname{deg} A_{n}}\right)^{t_{M}}} \mu\left(I\left(A_{1}, \ldots, A_{n-1}\right)\right) .
$$

It is easy to check that the measure $\mu$ is well defined, by (11) we have:

$$
\sum_{A_{n+1}} \mu\left(I\left(A_{1}, A_{2}, \ldots, A_{n+1}\right)\right)=\mu\left(I\left(A_{1}, A_{2}, \ldots, A_{n}\right)\right), \text { and } \sum_{A_{1}} \mu\left(I\left(A_{1}\right)\right)=1 .
$$

Then by Kolmogorov extension theorem, the set function $\mu$ can be extended into a probability measure supported on $F(M, p)$.

\section{Part II. Estimate the measures of the admissible cylinders}

In this part, we estimate the measure on admissible cylinders and show (12) is true on them.

In fact, for $n>p$, denote $s=\left\lfloor\frac{n}{p}\right\rfloor$, i.e., the integer part of $\frac{n}{p}$, for any $\left(A_{1}, \ldots, A_{n}\right) \in D_{n}$

$$
\begin{aligned}
& \mu\left(I\left(A_{1}, \ldots, A_{n}\right)\right) \\
= & \left(\frac{1}{(q-1) q^{M}}\right)^{p} \frac{1}{(q-1) q} \frac{1}{q t_{M} \sum_{j=s p+2}^{n} \operatorname{deg} A_{j}} \prod_{k=1}^{s-1}\left(\frac{1}{(q-1) q}\right)^{2} \frac{1}{q t_{M} \sum_{j=2}^{p-1} \operatorname{deg} A_{k p+j}} .
\end{aligned}
$$

While

$$
\begin{aligned}
\left|I\left(A_{1}, \ldots, A_{n}\right)\right| & =q^{-2\left(\sum_{j=1}^{n} \operatorname{deg} A_{j}\right)} \\
& =\frac{1}{q^{2 M p}} \frac{1}{q^{2}}\left(\frac{1}{q^{2 \sum_{j=s p+2}^{n} \operatorname{deg} A_{j}}}\right) \prod_{k=1}^{s-1}\left(\frac{1}{q^{2}}\right)^{2}\left(\frac{1}{q^{2 \sum_{j=2}^{p-1} \operatorname{deg} A_{k p+j}}}\right) .
\end{aligned}
$$

So

$$
\frac{\mu\left(I\left(A_{1}, \ldots, A_{n}\right)\right)}{\left|I\left(A_{1}, \ldots, A_{n}\right)\right|^{t_{M}-\epsilon}}=J \cdot \prod_{k=1}^{s-1} J_{k}
$$

where

and

$$
J=\frac{\left(q^{2 M p}\right)^{t_{M}-\epsilon}}{\left((q-1) q^{M}\right)^{p}} \frac{q^{2\left(t_{M}-\epsilon\right)}}{(q-1) q}\left(\frac{1}{q^{2 \sum_{j=s p+2}^{n} \operatorname{deg} A_{j}}}\right)^{\epsilon}
$$

$$
J_{k}=\left(\frac{q^{2\left(t_{M}-\epsilon\right)}}{(q-1) q}\right)^{2}\left(\frac{1}{q^{2 \sum_{j=2}^{p-1} \operatorname{deg} A_{k p+j}}}\right)^{\epsilon} .
$$

Since $\epsilon \geq 0$ and $\operatorname{deg} A_{j} \geq 1$, then

$$
J \leq \frac{\left(q^{2 M p}\right)^{t_{M}}}{\left((q-1) q^{M}\right)^{p}} \frac{q^{2 t_{M}}}{(q-1) q}:=C
$$

and

$$
J_{k} \leq\left(\frac{q^{2\left(t_{M}-\epsilon\right)}}{(q-1) q}\right)^{2} \frac{1}{q^{2 \epsilon(p-2)}}=1
$$


where the last equality is from (13). Combining (17), we have

$$
\mu\left(I\left(A_{1}, \ldots, A_{n}\right)\right) \leq C\left|I\left(A_{1}, \ldots, A_{n}\right)\right|^{t_{M}-\epsilon} .
$$

Part III. Estimate the measures of arbitrary balls

In this part, we estimate the measure $\mu$ on an arbitrary ball $B(x, r)$ and show (12) is true, which implies the result of Lemma 4.4 by Mass Distribution Principle (Proposition 4.3).

Let $B(x, r)$ be a ball with center $x \in I$. We can assume $x \in F(M, p)$. Otherwise, $B(x, r) \cap F(M, p)=\emptyset$, then $\mu(B(x, r))=0$ since $\mu$ is supported on $F(M, p)$, thus (12) will hold trivially. We denote by $I\left(A_{1}, \ldots, A_{n}\right)$ the $n$-th admissible cylinder containing $x$. For any $r$ small enough, there exists $n \in \mathbb{N}$ such that

$$
\left|I\left(A_{1}, \ldots, A_{n}, A_{n+1}\right)\right|<r \leq\left|I\left(A_{1}, \ldots, A_{n}\right)\right| .
$$

Then by Remark 1.1, it follows

$$
I\left(A_{1}, \ldots, A_{n}, A_{n+1}\right) \subset B(x, r) \subset I\left(A_{1}, \ldots, A_{n}\right) .
$$

And by the construction of the measure $\mu$ and the result of Part II (see (14), (15), (18)), denote $C^{\prime}=\max \left\{q(q-1), q^{2 M t_{M}}\right\}$, then

$$
\begin{aligned}
\mu(B(x, r)) & \leq \mu\left(I\left(A_{1}, \ldots, A_{n}\right)\right) \leq C^{\prime} \mu\left(I\left(A_{1}, \ldots, A_{n}, A_{n+1}\right)\right) \\
& \leq C^{\prime} C\left|\left(I\left(A_{1}, \ldots, A_{n+1}\right)\right)\right|^{t_{M}-\epsilon} \leq C^{\prime} C r^{t_{M}-\epsilon} .
\end{aligned}
$$

Therefore (12) holds for any ball.

\subsection{Proof of Theorem 1.3}

Before we prove the last theorem, we give the following lemma. Let $x \in$ I. Denote by $I_{k}(x)=I\left(A_{1}(x), \ldots, A_{k}(x)\right)$ the admissible cylinder of order $k$ containing $x$ and by $R_{k}(x)=\inf \left\{n \geq 1: T^{n}(x) \in I_{k}(x)\right\}$ the first return time of $x$ into $I_{k}(x)$.

Lemma 4.6. For each $x \in F(M)$, we have

$$
\underline{R}(x)=\liminf _{k \rightarrow \infty} \frac{\log _{q} R_{k}(x)}{-\log \left|I_{k}(x)\right|}, \quad \bar{R}(x)=\limsup _{k \rightarrow \infty} \frac{\log _{q} R_{k}(x)}{-\log \left|I_{k}(x)\right|},
$$

Proof. For each ball $B\left(x, q^{-m}\right)$, we can find $k \in \mathbb{N}$ such that

$$
I\left(A_{1}(x), \ldots, A_{k+1}(x)\right) \subset B\left(x, q^{-m}\right) \subset I\left(A_{1}(x), \ldots, A_{k}(x)\right),
$$

which means $R_{k}(x) \leq \tau_{q^{-m}}(x) \leq R_{k+1}(x)$. The equation (20) implies that

$$
q^{-2\left(\operatorname{deg} A_{1}(x)+\cdots+\operatorname{deg} A_{k+1}(x)\right)} \leq q^{-m} \leq q^{-2\left(\operatorname{deg} A_{1}(x)+\cdots+\operatorname{deg} A_{k}(x)\right)},
$$

then

$$
\frac{\log _{q} R_{k}(x)}{-\log _{q}\left|I_{k+1}(x)\right|} \leq \frac{\log _{q} \tau_{q^{-m}}(x)}{m} \leq \frac{\log _{q} R_{k+1}(x)}{-\log _{q}\left|I_{k}(x)\right|}
$$

Thus the fact that $x \in F(M)$ implies (19). 
Proof of Theorem 1.3. Let $\left\{n_{k}\right\}$ be the sequence satisfying the following two conditions (we will give the detail choice of the sequence in the following):

(a) $\exists k_{0}$, such that $n_{k+1} \geq n_{k}+2 k$ for any $k \geq k_{0}$;

(b) $\lim _{k \rightarrow \infty} n_{k} / k^{3}=+\infty$.

For any $\delta>0$, by Proposition 4.2 , there exists $M_{0} \in \mathbb{N}$ such that

$$
t_{M} \geq 1-\delta / 2
$$

for any $M \geq M_{0}$. We can choose an integer $p$ satisfying

$$
p \geq \max \left\{\frac{4-2 \log _{q} q(q-1)}{\delta}, k_{0}\right\} \text {. }
$$

By (21), we know $\epsilon:=\frac{2 t_{M}-\log _{q} q(q-1)}{p} \leq \frac{2-\log _{q} q(q-1)}{p} \leq \frac{\delta}{2}$ for any $M \geq M_{0}$. In the light of Lemma 4.4 , for any $M \geq M_{0}$, we have

$$
\operatorname{dim}_{H} F(M, p) \geq t_{M}-\epsilon \geq 1-\frac{\delta}{2}-\frac{\delta}{2} \geq 1-\delta .
$$

For each $x \in F(M, p)$, let $\left[A_{1}^{0}, \ldots, A_{n}^{0}, \ldots\right]$ be its continued fraction expansion, we will construct $x^{*} \in I$ by induction using the main idea of [7] in symbolic spaces.

Let $x^{(0)}=x^{(1)}=\cdots=x^{(p-1)}=\left[A_{1}^{0}, \ldots, A_{n}^{0}, \ldots\right]=x$. For any $k \geq p$, we construct $x^{(k)}$ from $x^{(k-1)}$ by inserting the block $A A_{1}^{(k-1)} \cdots A_{k}^{(k-1)} B_{k} A$ of length $k+3$ at the position $n_{k}$, i.e.,

$$
x^{(k)}=\left[A_{1}^{(k-1)}, \ldots, A_{n_{k}-1}^{(k-1)}, A, A_{1}^{(k-1)}, \ldots, A_{k}^{(k-1)} B_{k}, A, A_{n_{k}}^{(k-1)}, \ldots\right],
$$

where $A$ is a fixed polynomial with $\operatorname{deg} A=1$ and $B_{k}$ is any polynomial with $B_{k} \neq A_{k+1}^{(k-1)}$.

We shall choose $n_{k}$ to be different quantity according to the different value of $\alpha$ and $\beta$ by using the results of lemma 4.6.

CASE 1: $\alpha=\beta=\infty$, let $n_{k}=\left\lfloor q^{k^{2} \cdot M}\right\rfloor$, here and afterwards $\lfloor x\rfloor$ denotes the integral part of $x$.

CASE 2: $0<\alpha=\beta<\infty$, let $n_{k}=\left\lfloor\left|I_{k}(x)\right|^{-\alpha}\right\rfloor$.

CASE 3: $\alpha=\beta=0$, let $n_{k}=\left\lfloor q^{\sqrt{k}}\right\rfloor$.

CASE 4: $0<\alpha<\beta<\infty$, if $\sum_{j=1}^{2 i-1} 2^{4^{j}} \leq k \leq \sum_{j=1}^{2 i} 2^{4^{j}}$ for some $i \geq 0$, define $l_{k}=\left\lfloor\left|I_{k}(x)\right|^{-\alpha}\right\rfloor$, otherwise, let $l_{k}=\left\lfloor\left|I_{k}(x)\right|^{-\beta}\right\rfloor$. Choose $n_{k}=\sum_{i=1}^{k} l_{i}$.

CASE 5: $0<\alpha<\beta=\infty$, if $\sum_{j=1}^{2 i-1} 2^{4^{j}} \leq k \leq \sum_{j=1}^{2 i} 2^{4^{j}}$ for some $i \geq 0$, define $l_{k}=\left\lfloor\left|I_{k}(x)\right|^{-\alpha}\right\rfloor$, and otherwise, let $l_{k}=\left\lfloor q^{k^{2} \cdot M}\right\rfloor$. Choose $n_{k}=\sum_{i=1}^{k} l_{i}$.

CASE 6: $0=\alpha<\beta<\infty$, if $\sum_{j=1}^{2 i-1} 2^{4^{j}} \leq k \leq \sum_{j=1}^{2 i} 2^{4^{j}}$ for some $i \geq 0$, define $l_{k}=\left\lfloor q^{\sqrt{k}}\right\rfloor$, otherwise, let $l_{k}=\left\lfloor\left|I_{k}(x)\right|^{-\beta}\right\rfloor$. Choose $n_{k}=\sum_{i=1}^{k} l_{i}$. 
CASE 7: $0=\alpha, \beta=\infty$, if $\sum_{j=1}^{2 i-1} 2^{4^{j}} \leq k \leq \sum_{j=1}^{2 i} 2^{4^{j}}$ for some $i \geq 0$, define $l_{k}=\left\lfloor q^{\sqrt{k}}\right\rfloor$, otherwise, let $l_{k}=\left\lfloor q^{k^{2} \cdot M}\right\rfloor$ otherwise. Choose $n_{k}=\sum_{i=1}^{k} l_{i}$.

For above all the cases, we note that the increasing speed of $n_{k}$ is at least exponential order, which surely satisfies the conditions (a) and (b).

It is easy to check that the first $n_{k}-1$ digits of $x^{(k)}$ and $x^{(k-1)}$ coincide by the condition of $(a)$, which leads to the Cauchy properties of sequence $\left\{x^{(k)}\right\}$. Since the field $\mathbb{F}_{q}\left(\left(X^{-1}\right)\right)$ is a complete metric space, we denote by $x^{*}$ the limit of $\left\{x^{(k)}\right\}$. Denote by $F$ the set of points $x^{*}$ determined by $x \in F(M, p)$. From the construction of $x^{*}$, we have $R_{k}\left(x^{*}\right)=n_{k}$. By the construction of $\left\{n_{k}\right\}$, we know $\underline{R}(x)=\alpha$ and $\bar{R}(x)=\beta$. Therefore, $F \subset E_{\alpha, \beta}$.

Next we define a function $h: F(M, p) \rightarrow F$ by $x \mapsto x^{*}$. It is easy to find that $h$ is injective. We will show that $h^{-1}$ is Hölder continues on the image $h(F(M, p))$.

Assume that $\left\|x^{*}-y^{*}\right\| \leq q^{-k}$. For any $k$ large enough, there exists $n \in \mathbb{N}$ such that

$$
\left|I\left(A_{1}\left(x^{*}\right), \ldots, A_{n+1}\left(x^{*}\right)\right)\right|<q^{-k} \leq\left|I\left(A_{1}\left(x^{*}\right), \ldots, A_{n}\left(x^{*}\right)\right)\right| .
$$

It follows

$$
y^{*} \in I\left(A_{1}\left(x^{*}\right), \ldots, A_{n}\left(x^{*}\right)\right) .
$$

This means $A_{1}\left(x^{*}\right)=A_{1}\left(y^{*}\right), \ldots, A_{n}\left(x^{*}\right)=A_{n}\left(y^{*}\right)$. Let $w$ be the integer with $n_{w} \leq n \leq n_{w+1}$. Thus

$$
A_{1}(x)=A_{1}(y), \ldots, A_{n^{\prime}}(x)=A_{n^{\prime}}(y)
$$

by the construction of $x^{*}$ and $y^{*}$ and $n^{\prime}=n-\sum_{j=1}^{w}(j+3)$. Therefore,

$$
\begin{aligned}
\|x-y\| & \leq\left|I\left(A_{1}(x), \ldots, A_{n^{\prime}}(x)\right)\right|=q^{-2 \sum_{k=1}^{n^{\prime}} \operatorname{deg} A_{k}(x)} \\
& \leq q^{-2 \sum_{k=1}^{n+1} \operatorname{deg} A_{k}\left(x^{*}\right)} \cdot q^{2 M\left(n-n^{\prime}\right)} \\
& \leq q^{-k} \cdot q^{2 M\left(n-n^{\prime}\right)} .
\end{aligned}
$$

By the condition of $(b)$, for any $\epsilon>0$, there exists an integer $N>p$ such that $k^{3} / n_{k}<\epsilon / 2$ for all $k \geq N$. Then

$$
\begin{aligned}
2 M\left(n-n^{\prime}\right) & \leq 2 M \sum_{j=1}^{w}(j+3) \leq 4 M w^{2} \leq 2 \epsilon M \frac{n_{w}}{w} \leq 2 \epsilon\left(n \cdot \frac{M}{w}\right) \\
& =2 \epsilon\left(\frac{M}{w}+\cdots+\frac{M}{w}\right) \leq 2 \epsilon\left(\operatorname{deg} A_{1}(x)+\cdots+\operatorname{deg} A_{n}(x)\right) \\
& \leq k \epsilon .
\end{aligned}
$$

Thus

$$
\|x-y\| \leq q^{-k(1-\epsilon)}
$$


By the properties of Hölder continuous function about Hausdorff dimension (see Proposition $2.3[6]$ ), we have

$$
\operatorname{dim}_{H} h(F(M, p)) \geq \frac{1}{1-\epsilon} \operatorname{dim}_{H} F(M, p) .
$$

By the arbitrariness of $\epsilon$, we have

$$
\operatorname{dim}_{H} F \geq \operatorname{dim}_{H} h(F(M, p)) \geq \operatorname{dim}_{H} F(M, p) \geq 1-\delta .
$$

This completes the proof of Theorem 1.3 by the arbitrariness of $\delta$ and $F \subset$ $E_{\alpha, \beta}$.

Acknowledgement. The authors thank the referee for the helpful comments and Prof. Jun Wu for his constant encouragements. This work was supported by NSFC 11001093, 10901066, 11101167, 11126071; the Project-sponsored by SRF for ROCS, SEM; and 'the Fundamental Research Funds for the Central Universities' SCUT: 2012ZB0018, 2012ZZ0073 and 2013ZZ0085, HUST: 2013TS140, HZAU:2013PY116.

\section{References}

[1] E. Artin, Quadratische Körper im Gebiete der höheren Kongruenzen, I-II, Math. Z. 19 (1924), no. 1, 153-246.

[2] L. Barreira and B. Saussol, Hausdorff dimension of measures via Poincaré recurrence, Comm. Math. Phys. 219 (2001), no. 2, 443-463.

[3] _ Product structure of Poincare recurrence, Ergodic Theory Dynam. Systems 22 (2002), no. 1, 33-61.

[4] V. Berthé and H. Nakada, On continued fraction expansions in positive characteristic: equivalence relations and some metric properties, Expo. Math. 18 (2000), no. 4, 257284 .

[5] M. Boshernitzan, Quantitative recurrence results, Invent. Math. 113 (1993), no. 3, 617631.

[6] K. J. Falconer, Fractal Geometry, Mathematical Foundations and Application, Wiley, 1990.

[7] D. J. Feng and J. Wu, The Hausdorff dimension of recurrent sets in symbolic spaces, Nonlinearity 14 (2001), no. 1, 81-85.

[8] X. H. Hu, B. W. Wang, J. Wu, and Y. L. Yu, Cantor sets determined by partial quotients of continued fractions of Laurent series, Finite Fields Appl. 14 (2008), no. 2, 417-437.

[9] K. S. Lau and L. Shu, The spectrum of Poincare recurrence, Ergodic Theory Dynam. Systems 28 (2008), no. 6, 1917-1943.

[10] H. Niederreiter, The probabilistic theory of linear complexity, Advances in cryptologyEUROCRYPT '88 (Davos, 1988), 191209, Lecture Notes in Comput. Sci., 330, Springer, Berlin, 1988.

[11] H. Niederreiter and M. Vielhaber, Linear complexity profiles: Hausdorff dimensions for almost perfect profiles and measures for general profiles, J. Complexity. 13 (1997), no. 3, 353-383.

[12] D. Ornstein and B. Weiss, Entropy and data compression schemes, IEEE Trans. Inform. Theory 39 (1993), no. 1 78-83.

[13] R. Paysant-Leroux and E. Dubois, Étude métrique de l'algorithme de Jacobi-Perron dans un corps de séries formelles, (French) C. R. Acad. Sci. Paris Sér. A-B 275 (1972), A683-A686. 
[14] B. Saussol and J. Wu, Recurrence spectrum in smooth dynamical system, Nonlinearity 16 (2003), no. 6, 1991-2001.

[15] W. M. Schmidt, On continued fractions and Diophantine approximation in power series fields, Acta Arith. 95 (2000), no. 2, 139-166.

[16] J. Wu, Hausdorff dimensions of bounded type continued fraction sets of Laurent series, Finite Fields Appl. 13 (2007), no. 1, 20-30.

Xue-Hai Hu

College of Science

Huazhong Agricultural University

Wuhan, Hubei, 430070, P. R. China

E-mail address: jackyhuxuehai@hotmail.com

BING LI

Department of Mathematics

South China University of Technology

510640, Guangzhou, P. R. China

E-mail address: libing0826@gmail.com

JIAN XU

School of Mathematics and Statistics

Huazhong University of Science And Technology

Wuhan, Hubei, 430074, P. R. China

E-mail address: arielxj@hotmail.com 